\title{
Clinical and Dermoscopic Features of a Rare Histologic Variant: Acantholytic Squamous Cell Carcinoma
}

\section{Senhaji G*, El Jouari 0, Gallouj S, Douhi Z, Bay HB and Mernissi FZ \\ Department of Dermatology, University Hospital Hassan II Fez, Morocco}

*Corresponding author: Senhaji Ghita, Doctor, Department of Dermatology, University hospital Hassan II, Fez, Morocco, Tel: +212 600075857; Email: ghitasenhaji88@gmail.com

\section{Case Report \\ Volume 3 Issue 2}

Received Date: September 24, 2018

Published Date: November 05, 2018

DOI: $10.23880 /$ cdoaj- 16000155

\section{Abstract}

Acantholytic squamous cell carcinoma (ASCC) is an uncommon variant of squamous cell carcinoma (SCC), in which significant portions of the neoplastic proliferation show a pseudoglandular or tubular microscopic pattern. It's a rare tumor representing 2 to $4 \%$ of all cutaneous SCC, and it is usually found on the sun exposed areas of elderly patients with a notable male predominance. The prognosis of this variant is matter of debate, although it appears to behave more aggressively than non-acantholytic, well-differentiated SCC. We report a rare case of a 92 year old female patient presenting with an ASCC of the left cheek emphasizing the dermoscopic signs found, that are missing in the literature.

Keywords: Acantholytic squamous cell carcinoma; Squamous cell carcinoma; Dermoscopy; Pseudoglandular squamous cell carcinoma

\section{Introduction}

Acantholytic squamous cell carcinoma (ASCC) is an uncommon variant of squamous cell carcinoma (SCC) [1], in which significant portions of the neoplastic proliferation show a pseudoglandular or tubular microscopic pattern [2]. This variant is also known as adenoid SCC, adenoacanthoma of sweat glands, and pseudoglandular SCC [3], and few cases have been reported in literature [4]. The most frequent locations of the tumor are the sun exposed ones, however many other different sites have been reported [5]. The prognosis of ASCC is matter of debate [2], although it appears to behave more aggressively than non-acantholytic, welldifferentiated SCC [6]. We report a rare case of a 92 year old female patient presenting with an ASCC of the left cheek.

\section{Case Report}

A 92-year-old woman, with a history of chronic and intermittent solar exposure in childhood, presented to our Dermatology department with a 6 months history of a solitary painful, erythematous and well demarked nodule, in the left cheek. This nodule increased rapidly in size after chronic and repetitive manipulation. There was no previous personal or familial history of skin cancer and no other significant medical history was elicited.

Clinical examination revealed a solitary, erythematous and well-defined tumor of firm consistency, with smooth surface, measuring $2.5 \mathrm{~cm}$ at long axis and located at the left cheek, surrounded by multiple actinic keratosis (Figure 1). 


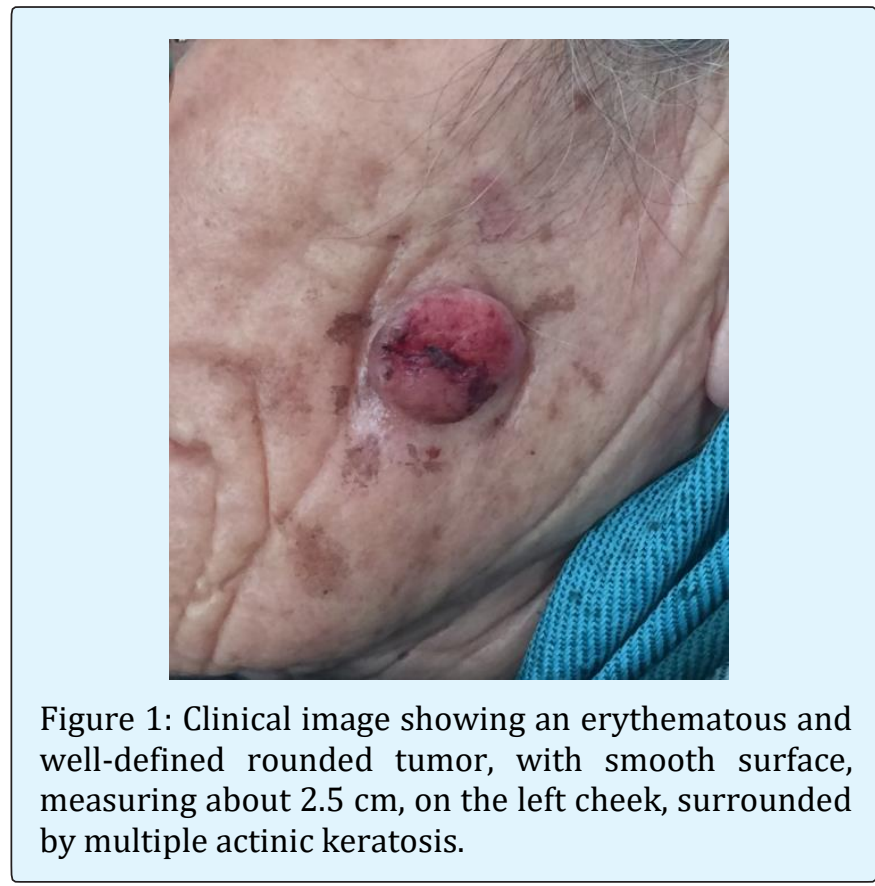

Dermoscopy showed a yellowish background along with erythematous areas, and polymorphic vascularization made of linear irregular and hairpin vessels, more marked at the periphery of the lesion with hemorrhagic suffusions (Figure 2). The local neurological examination was normal and no lymphadenopathy was appreciated. Clinically, achromic melanoma, basal cell carcinoma and squamous cell carcinoma were suspected, and a skin biopsy was performed to make the diagnosis. Histologic examination confirmed the acantholytic variant of SCC by showing marked acantholysis associated with dissociation of tumor cells made from cuboid cells having a basophilic cytoplasm with pseudoglandular cavity formations (Figure 3). The immunohistochemical complement showed cells reactive with anti P63 and anti Ck5 / 6 antibodies (Figure 4), while they were negative for anti Ck 7, 20, 19 and to anti ACE. Based on clinical and histopathological findings, this case was diagnosed as an ASCC. An extension assessment made of cervico-facial CT had returned without abnormalities, then the patient was referred in maxillofacial surgery for excision of the entire lesion with safety margins. No recurrence was noted for 18 months.

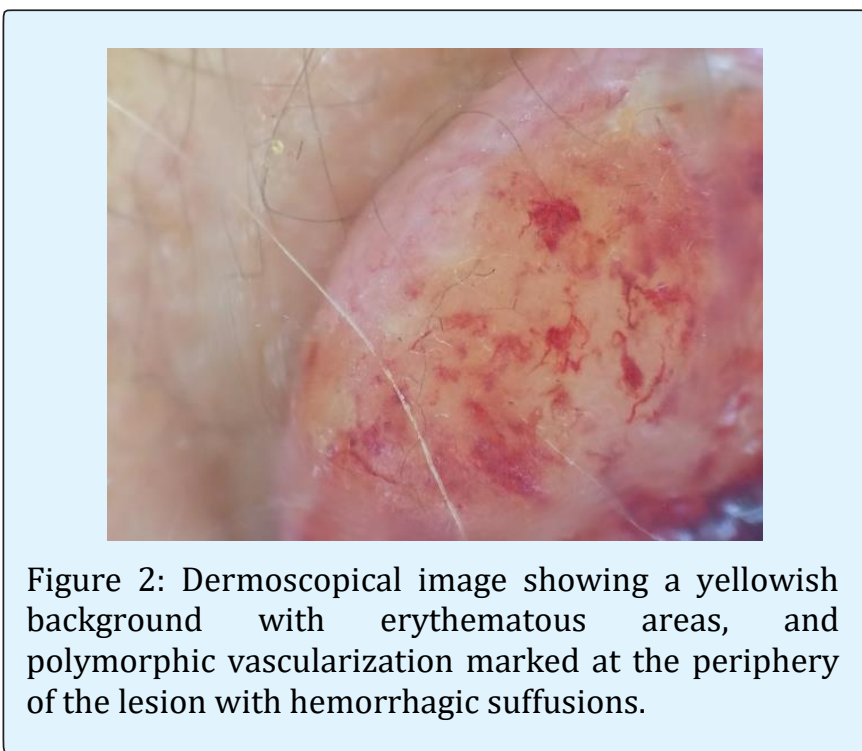

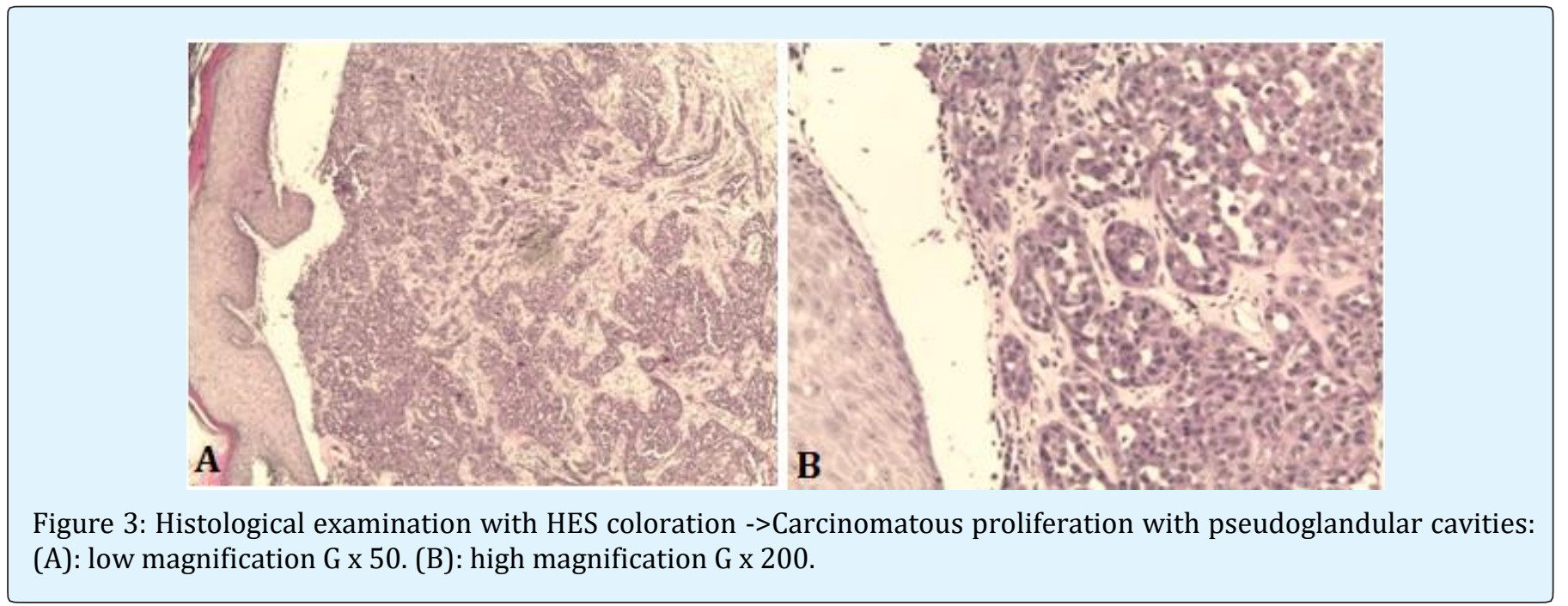



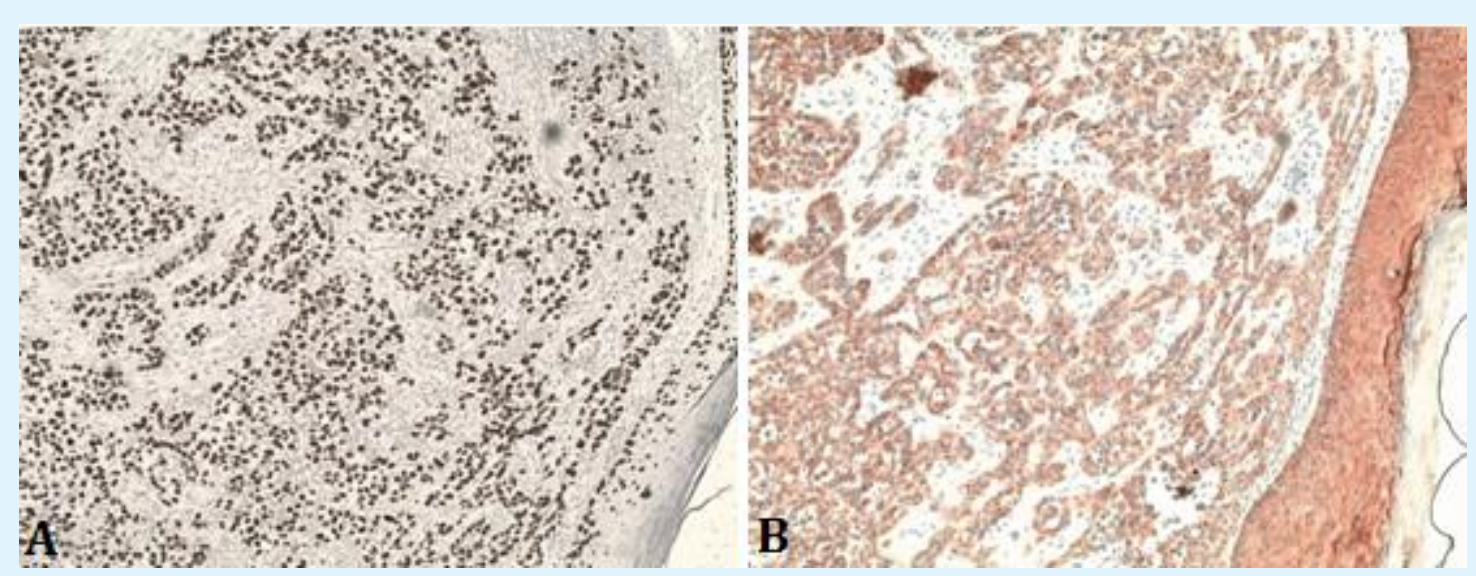

Figure 4: IHC Coloration G x 100->(A): P63: Diffuse nuclear marking. (B): CK5/6: Diffuse marking of tumor proliferation.

\section{Discussion}

Initially described in 1947 by Lever, the ASCC was considered to have a sweat gland origin, and was named adenoacanthoma of sweat glands [5]. However, later it was accepted as an uncommon variant of squamous cell carcinoma [4]. It represents $2-4 \%$ of all cutaneous squamous cell carcinoma [5], and it is usually found on the sunexposed areas of elderly patients with notable male predominance [2]. However, an increased incidence of acantholytic SCC has been recorded in organ transplant patients [6].The lesions typically arise on the head and neck as a nodule or ulcer, particularly on and around the ears and face, de novo or may develop from an actinic keratosis [5], although other less-sun-exposed locations have been reported [1], including the vulva, penis, oral mucosa, nasopharynx, and breast [2].

Clinicaly, it presents as a nodular, flesh colored to red, scaling, crusted, and ulcerated lesion [1], with a slow growing pattern [5]. Therefore, histological examination is necessary for making the accurate diagnosis [2].Histological characteristics include typical squamous differentiation with discrete or diffuse areas of keratinocytic acantholysis with a pseudoglandular appearance [1]. In fact, the lesional keratinocytes show a variable degree of desmosomal disruption [3], resulting in rounded free-floating single cells or small clusters of cells [6], arranged in tubular and gland-like structures, which is referred to as a "pseudoglandular" appearance [2]. Moreover, at high-power field, we can identify the malignant criteria of cells, including voluminous, hyperchromatic and pleomorphic nuclei presenting atypical mitosis [5]. In immunohistochemistry, this tumor resembles the structure of eccrine neoplasms, but it is negative for dPAS, CEA and mucicarmine and it is only positive for EMA and cytokeratins [2].

The differential diagnoses for acantholytic SCC include adenoid BCC, eccrine carcinoma, metastatic adenocarcinoma, and, rarely, angiosarcoma [3]. BCC must show, at least in part, the typical peripheral palisading, peritumoral lacunae, and stromal mucin [2]. Identifying ductal structures with a basal or myoepithelial layer that stains for smooth muscle actin, p63, calponin, or S100 protein, luminal borders that stain for carcinoembryonic antigen, and luminal secretions that stain with periodic acid-Schiff distase help to distinguish the eccrine carcinoma [3]. Metastatic adenocarcinoma shows multiplicity, acantholytic dyskeratosis and the absence of clear epidermal attachments [2]. While angiosarcoma can be suspected from blood-filled spaces and confirmed with various endothelial markers such as CD31, CD34, and ERG [3].

There are no specific risk factors for ASCC, however, it conserves the same risk factors as regular SCC, such as ultraviolet lights and radiation therapy [5]. It also has been documented to arise in areas of pre-existing scarring and in patients with chronic lymphocytic leukemia [2].

The treatment for ASCC is usually the same as for conventional SCC, and is based mainly on surgical excision, which may be followed by skin graft for deep invaded skin tumors [5]. 


\section{Clinical Dermatology Open Access Journal}

There is no comprehensive study on the biologic behavior of ASCC [1], and it prognosis of is matter of debate [2]. It is often regarded as having greater potential for metastasis and recurrence, despite the paucity of data to confirm this conclusion [1]. Additionally, its behavior appears to be depth dependent [5]. Of the articles investigated, the recurrence and metastasis rates ranged from $6 \%$ to $10 \%$ to $2 \%$, to $43 \%$, respectively, which is comparable to data published for regular SCC [1]. Furthermore, to date, there is no uniform evidence that acantholytic SCC is more aggressive than non-acantholytic SCC [6].

\section{Conclusion}

ASCC is a rare histopathological variety of SCC, which frequently develops on sun exposed regions of skin, usually at elderly on preexisting lesions such as solar keratosis [5]. It has a characteristic 'pseudoglandular' appearance, so making the differential diagnosis via immunohistochemistry to exclude other malignant tumors may be important [2]. The prognosis of this rare entity is still in debate and has not been established yet. Thus, reports of more cases would possibly help us to understand more these rare tumors and to elucidate their clinical behavior.

\section{References}

1. Garcia C, Crowson AN (2011) Acantholytic squamous cell carcinoma: is it really a more-aggressive tumor? Dermatol Surg 37(3): 353-356.

2. Lim JY, Do MO, Kim SH, Hahm JH, Whang KK (2008) A Case of Acantholytic Squamous Cell Carcinoma. Ann Dermatol 20(4): 267-270.

3. Parekh V, Seykora JT (2017) Cutaneous Squamous Cell carcinoma. Clin Lab Med 37(3): 503-525.

4. Alhumidi A (2013) Acantholytic squamous cell carcinoma arising in a nevus sebaceous: A case report. Int J Health Sci (Qassim) 7(3): 343-346.

5. Sajin M, Hodorogea Prisăcaru A, Luchian MC, Pătraşcu OM, Dumitru A, et al. (2014) Acantholytic squamous cell carcinoma: pathological study of nine cases with review of literature. Rom J Morphol Embryol 55(2): 279-283.

6. Pyne JH, Myint E, Barr EM, Clark SP, David M, et al. (2017) Acantholytic invasive squamous cell carcinoma: Tumor diameter, invasion depth, grade of differentiation, surgical margins, perineural invasion, recurrence and death rate. J Cutan Pathol 44(4): 320327.

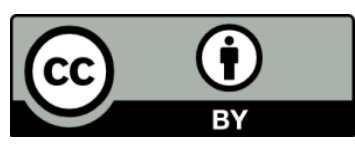

